\title{
Deutschwerden in Zafer Șenocaks Werken
}

\begin{abstract}
Im Beitrag wird das Engagement des deutschen Schriftstellers Zafer Şenocak für die Anerkennung eines neudeutschen Deutschland, das nicht mehr nur ethnisch gebunden wäre, beleuchtet. Der eigentliche Bruchpunkt in seiner Biografie war keineswegs die eigene Migrationserfahrung, sondern die Wende und die damit einhergehende Suche nach einer neuen deutschen Identität. Zu dem sich ab den 1990er Jahren abzeichnenden Migrantisierungsprozess kamen infolge der Attentate vom 11. September 2001 und der wachsenden Polarisierung zwischen der westlichen und der moslemischen Welt religiöse Stigmatisierung und Essenzialisierung hinzu. Gegen diesen Aus- und Abgrenzungstrend bemüht er die Werte des deutschen Humanismus, der deutschen Aufklärung und der türkischen Mystik. Ein aufklärerisches Engagement für ein deutsches Neuland fordert er sowohl von den Deutschen als auch von den türkischen Einwanderern. Das homogene Deutschsein soll nun dem hybriden Deutschwerden weichen.
\end{abstract}

Keywords: Einwanderung; Deutschwerden; Vielvölkerrepublik; Hybridisierung; Transkulturalität; Willensliteratur

„,Deutschsein“ erschwert ,Deutschwerden“ erheblich“, stellt Zafer Șenocak im Essayband Zungenentfernung. Bericht aus der Quarantänestation (2001, 45) fest. Anhand seines literarischen, essayistischen und journalistischen Werks soll der Begriff ,Deutschwerden“ erörtert werden, es handelt sich um ein im Zuge der Arbeitsmigration ab den 1960er Jahren brisantes Thema (vgl. Șenocak 2001, 57). Vom Politischen ist nämlich die Sprache der Migration, die Sprache des ,deutschsprachigen Schriftstellers nicht deutscher Sprache‘, nicht wegzudenken. Darum gilt es auch Șenocaks politisch-gesellschaftliches Engagement zu untersuchen.

Der Begriff ,Deutschwerden' steht im Widerspruch zum üblichen Terminus ,Deutschsein', der die deutsche Identität als etwas Homogenes und Endgültiges definiert. Er eröffnet neue Perspektiven und betrifft sowohl die Hinzugekommenen als auch die Deutschen, weil er der wachsenden Hybridisierung in Deutschland gerecht wird. ,Deutsch werden“ kann nun auch derjenige, dessen Geschichte anfangs nicht deutsch geprägt ist. Früher war er aufgrund des Abstammungsprinzips, das seit 1913 in Deutschland galt, aus diesem Deutschwerdungsprozess ausgeschlossen, war kein Deutscher. Mit der Reform des deutschen Staatsbürgerschaftsrechts im Jahre 2000, d. h. der Ergänzung des Abstammungsprinzips durch das Territorialprinzip, und mit der Abschaffung der Optionspflicht für die Ausländerkinder und der Einführung des Doppelpasses im Jahre 2014 kann er

2 Open Access. (c) 2021 Marie-Noëlle Faure, published by De Gruyter. (c) BY-NC-ND This work is licensed under the Creative Commons Attribution-NonCommercial-NoDerivatives 4.0 International License. 
jetzt aber Deutscher werden. ,Deutschwerden“ verweist aber auch auf den Wandel von der Identität der Deutschen selbst, die im Zuge der Einwanderung und der Globalisierung, der „Entgrenzung und der Denationalisierung“ (Șenocak 2001, 57), und der daraus entstehenden Heterogenität und Hybridisierung nicht mehr nur orts-, nations- und kulturgebunden ist. Zwischen beiden Elementen, d. h. dem Fremden und dem Einheimischen, die früher strikt voneinander getrennt waren, besteht nun eine unleugbare Wechselwirkung. Daraus entsteht die Figur des ,Neuen Deutschen“, wie sie Zafer Șenocak in Deutschsein. Eine Aufklärungsschrift (2011), einem feurigen Plädoyer für die Neubestimmung des Deutschseins, nennt. Diese Bezeichnung wurde 2016 von Herfried und Marina Münkler zum Titel ihres Buches Die Neuen Deutschen: Ein Land vor seiner Zukunft gewählt, in dem sie die $\mathrm{Ab}$ - und Einwanderungswellen im deutschen Raum seit der pax augustana (Augsburger Religionsfrieden) zwischen Lutheranern und Katholiken aus dem Jahre 1555 und den eingeführten Prinzipien des eius regio, cuius religio (wessen Land, dessen Religion) und des ius emigrandi (Auswanderungsrecht) für andersgläubige Untertanen analysieren und die Aufnahme der ,Geflüchteten‘ seit 2015 als eine Chance für Deutschland bewerten.

1961 in Ankara geboren, kommt Zafer Șenocak erst mit acht Jahren nach Deutschland. Von Kind auf erlebte Zafer Șenocak, der aus dem kosmopolitischen Istanbul nach München umgezogen ist, die Mehrsprachigkeit als Bereicherung. Im Sammelband Deutschsein. Eine Aufklärungsschrift (2011) erzählt er, wie er die deutsche Sprache bei einer strengen, aber liebenswürdigen Bayerin lernte. Diese Frau hatte ihm vor allem die Liebe zu der deutschen Sprache beigebracht, hatte es geschafft, bei ihm eine emotionale Bindung an die deutsche Sprache zu erwecken: „Bei Frau Saal schmeckten die Wörter nach Kaffee und Kuchen, genauer gesagt, nach Apfelkuchen, der fast immer auf dem Tisch stand und von dem ich kosten durfte, wenn ich fleißig gewesen war.“ (Șenocak 2011, 13)

Bis zur Wiedervereinigung im Jahre 1990 hat Șenocak die frühere Umstellung vom Leben in der Türkei auf sein Leben in Deutschland nie als biografische Diskontinuität erlebt. ,Biografische Diskontinuität‘ erwartet man jedoch heute von einem so genannten ,Migrantenautor‘. Damals aber fiel seine Selbstwahrnehmung mit der Fremdwahrnehmung seiner selbst zusammen: Als junger deutscher Autor fühlte er sich im Münchener Literaturbetrieb völlig integriert. Keiner wäre auf die Idee gekommen, ihn zu fragen, warum er auf Deutsch schreibe, ob er auch auf Türkisch schreibe, wohin er in der Tat gehöre. Er galt als deutscher Autor. Dass er Deutsch sprach und schrieb, war nicht nur für ihn, sondern auch für die Außenstehenden eine Selbstverständlichkeit.

Als Wendepunkte in der Wahrnehmung Zafer Șenocaks durch die Deutschen gelten zwei weltgeschichtliche Ereignisse: erstens ,die Wende“ und zweitens die Terrorattentate vom 11. September 2001. 
Vor der Wende gingen die Deutschen mit ihrer schwer belasteten Vergangenheit und mit dem Thema Identität eher vorsichtig um. Wenn die „ausländischen Mitbürger“ (Șenocak 1993, 9) ${ }^{1}$ aufgrund des damals ausschließlich geltenden Blutrechts auch nicht ,Deutsch werden' durften, so hatten sie es um einiges leichter: „[Sie] teilten die Gegenwart Deutschlands mit den Deutschen, nicht jedoch ihre Geschichte“ (Șenocak 1993, 20). In der Bundesrepublik, die sich immer wieder darum bemühte, sich von der Last der nationalen Symbole zu befreien, war der Nationalismus ein Tabu. Das kam aber den Einwanderern zugute: Die Deutschen, die ihre Vergangenheit verdrängten und den Nationalismus verpönten, verhielten sich ihnen gegenüber eher gleichgültig, was ihnen erlaubte, sich, ohne großes Aufsehen zu erregen, in Deutschland niederzulassen. Für Șenocak wirkt sich aber heute die damals mangelnde Aufarbeitung der Vergangenheit auf die Herangehensweise an die Ausländerfrage sehr negativ aus.

Erst mit der Wende und der damit einhergehenden Rückbesinnung auf die Identität - „Spiegelein, Spiegelein an der Wand, wer ist am deutschsten im ganzen Land?“ (Șenocak, 1998, 90) - driften Selbst- und Fremdwahrnehmung auseinander. Die west- und ostdeutschen Brüder hatten zwar endlich zueinander gefunden, ihr Augenmerk galt aber dabei nicht den Hinzugekommenen und deren jahrzehntelangen Beiträgen zum Aufbau der deutschen Wirtschaft und Gesellschaft. Es entstand dann bei den Migranten der nicht unbegründete Eindruck, wegen der einheitsbedingten Politik vernachlässigt worden zu sein. Die Einwanderungspolitik rückte nämlich damals für ein Jahrzehnt in den Hintergrund. Der Roman Gefährliche Verwandtschaft (1998), in dem das Befremdetsein des Helden deutsch-türkischer Herkunft Sascha Muhteschem, der kurz nach der Wende aus den Vereinigten Staaten zurückkehrt, in einem nun vereinigten Berlin literarisiert wird, ist deshalb als Wenderoman wahrzunehmen. Mit dem Fall der Mauer geht für Sascha das Gefühl der Geborgenheit endgültig verloren. An die Stelle der Geborgenheit tritt Identität, wenigstens Identitätssuche, an die Stelle der Berliner Mauer treten „unsichtbare Mauern“ (Șenocak 1998, 47) auf dem nationalidentitären Weg. Der Schock war umso größer, als Șenocak, dessen Biografie Ähnlichkeiten mit derjenigen Saschas aufweist, 1989 schon über 22 Jahre in Deutschland lebte.

1 Zum Ausländergesetz in den 1990er Jahren schreibt er: „Die Diskussion über das neue Ausländergesetz in der Bundesrepublik [...] wurde weitgehend an uns, der zweiten Generation der türkischen Immigranten, vorbeigeführt. Denn unsere Realität wird in diesem Gesetz weiterhin missachtet. Wir können uns als hier Geborene und Aufgewachsene wohl kaum mit dem Begriff ,ausländische Mitbürger ‘ identifizieren, mit dem im vorliegenden Gesetz operiert wird.“ 
Das Ausgrenzungsgefühl, das Gefühl, nicht hierher zu gehören, steigerte sich vor allem bei der türkischen Gemeinschaft, auch bei denjenigen, denen das Herkunftsland oder das ihrer Eltern nicht so viel bedeutete. Nach den Brandanschlägen in Mölln und Solingen in den früheren 1990er Jahren wurden sich nämlich viele Türken ihrer Andersartigkeit - im Blick des Anderen - bewusst. Schuld an der Polarisierung ist Șenocak zufolge die lückenhafte bundesdeutsche Erinnerungskultur: Des Gastarbeiters werde überhaupt nicht gedacht. Er gehöre nicht ins bundesdeutsche Narrativ, als bliebe er ein Fremdkörper. (Vgl. Șenocak 2014)

Identitätsbildung durch Ausgrenzung des Anderen: Das blieb auch Zafer Șenocak als Autor nicht erspart. Über Nacht avancierte der deutsche Autor zum Migrantenautor, wurde als ,deutschsprachiger Autor nicht deutscher Muttersprache“ wahrgenommen: „In wissenschaftlichen Darstellungen war ich nunmehr ein türkischer Schriftsteller, der geschickt mit der deutschen Sprache umging.“ (Șenocak 1998, 129). Der Akzent wurde immer mehr auf seine Abstammung gelegt. Er wurde sich seines Stigmas (Vgl. Goffman 2010) bewusst, der fremdländisch klingende Name verriet nun seinen türkischen Migrationshintergrund, der bei der Fremdwahrnehmung immer mehr an Gewicht gewann: „,Sind Sie Ausländer?' wurde ich gefragt, wenn ich meinen Namen buchstabierte. Früher buchstabierte ich ihn ohne diese Frage.“ (Șenocak 1998, 128). Wie er es 2012 in einer im jüdischen Museum veranstalteten Podiumsdiskussion zum Thema Meine Liebe $z$ u Deutschland formulierte, wurde er „,von der Zugehörigkeitsfrage eingeholt.“ Er wurde als Autor migrantisiert, sein Werk musste in eine Nischenliteratur, die ,Migrantenliteratur‘, gehören, als müssten oder könnten sich ,deutschsprachige Autoren nicht deutscher Muttersprache‘ nur mit der Migrationsthematik beschäftigen: „Doch schon bald wurde mir auf Lesungen die Frage gestellt, was denn meine Texte eigentlich mit dem Leben der Türken in Deutschland zu tun hätten. Warum schrieb ich denn nicht über die Erfahrungen der Gastarbeiter? Über Fremdenfeindlichkeit?“ (Șenocak 1998,107).

Șenocak hat sich deswegen immer wieder vor der Exotismusfalle, der Orientalisierung des Orients in Acht genommen: „[Die Literatur des Orients] besteht bis in unsere Tage aus Märchen. Ein arabischer Autor ist immer nur ein Märchenerzähler.“ (Șenocak 1993, 25). Deshalb kritisiert er an der Autorin Emine Sevgi Özdamar die Einbeziehung morgenländischer Kulturelemente in ihre Werke und vor allem das archaische Image, das sie von der Türkei vermittle und das die Vorurteile der ,Westlichen' stärke. Es entspreche den Wunschbildern der westlichen Bevölkerung und zementiere unbewusst die Spaltung zwischen der Mehrheitsgesellschaft und den Minderheiten.

Dabei übertrifft das Kollektive das Individuelle, die ,Ich-Identität‘ des Schriftstellers wird nämlich immer wieder als „Störfaktor“ empfunden (Șenocak 2001, 20). Der Schriftsteller leidet seinerseits unter dem herrschenden Kulturalismus, 
der davon ausgeht, Individuen und deren Eigenschaften würden sich zu einem homogenen Modell, zu einer Gruppenidentität summieren lassen: „Die Mehrheit [läuft] Gefahr, die Kultur der Minderheit als Einzelheit zu betrachten ohne zu differenzieren, folkloristische Aspekte überzubetonen und immer die ethnologische Brille aufzusetzen.“ (Șenocak1993, 25)

Voller Ironie schildert Șenocak in „Dichter gibt Auskunft. ein Telefoninterview“, wie er den Interviewer verwirrt, der nicht mehr in der Lage ist, ihn in eine klar definierbare Kategorie einzuordnen: „Ich habe das Gefühl, mit meinen Fragen nicht weiter zu kommen. Sie geben ausweichende Antworten. Ich kann Sie in den Antworten nicht erkennen.“ (Șenocak 2006, 214).

Die Biografie des Migrantenautors und dessen literarisches Schaffen werden meistens auf eine biografische Diskontinuität, auf den Bruchpunkt, d. h. das Verlassen der Heimat und den Aufbruch in die Fremde, reduziert, als könnte er nicht in der Fremde ankommen, als hätten seine Werke keinen Anspruch auf Literarizität, auf Kreativität: „Man liest nicht die Texte des Autors, sondern seine ihm auf den Leib geschriebene Biographie. Herkunft ersetzt die Biographie.“ (Șenocak 2001, 98). Eine Biografie ist laut Șenocak viel mehr als die reine Summe der Erlebnisse, ganz besonders bei einem Dichter, bei dem die Einbildungskraft eine bedeutende Rolle spielt. Sie besteht zwar aus dem Selbsterlebten, aber auch aus dem Erdachten und dem Erzählten, „das Fiktive macht die Substanz der dichterischen Biographien aus“ (Șenocak 2001, 92). Nicht nur verwirft er die Festlegung auf die Ethnie, sondern auch die auf ein geografisches Territorium. ,Jeder Mensch hat seine eigene persönliche Geographie, in der Grenzen anders verlaufen als auf der Landkarte“ (Șenocak 2001, 23). Die persönliche Geografie eines Menschen deckt sich nicht mit den geografischen Grenzen, die als Identitätsmarker oft zur Identitätsstiftung durch Abgrenzung beitrugen und immer noch beitragen.

Șenocaks Helden sind deswegen sehr oft mehrsprachig. In der Widmung seines Essaybands Deutschsein. Eine Aufklärungsschrift dankt er seinem Vater dafür, dass er ihn „gelehrt hat, dass Wurzeln mehrsprachig sind.“ (Șenocak 2011). Die Helden haben verschiedene, manchmal schwer miteinander zu vereinbarende Wurzeln. Deshalb wechselt der Schauplatz seiner Erzählungen nach Berlin, deshalb zog Șenocak selbst nach Berlin. Zwar erscheint die Stadt Berlin als die Hauptstadt eines identitätssuchenden wiedervereinigten Deutschland, sie gilt aber als die einzige kosmopolitische, ,aufnahmefähige“ Stadt in ganz Deutschland (Șenocak 2001, 23). Eine „atonale“ Stadt, in Anlehnung an Șenocaks Vergleich zwischen Einwanderungsland und atonaler Musik in seinem Essay Die atonale Welt. Wie viel Vielfalt ertragen wir? (Vgl. Șenocak 2001, 36-58). Eine atonale Stadt im ständigen Werden (vgl. Șenocak 2001, 23). Gerade dieses Atonale im ständigen Werden gilt als Modell für eine Neubestimmung des Deutschseins, das nicht mehr auf Muttersprache und ethnische Zugehörigkeit festgelegt werden 
soll. Das Atonale gilt sowohl auf nationaler Ebene, wie aus dem Titel des Sammelbands Atlas des tropischen Deutschland - in Anlehnung an die Gegenüberstellung des deutschen Waldes und des Tropenwaldes bei Elias Canetti ${ }^{2}-\mathrm{zu}$ entnehmen ist, als auch auf persönlicher Ebene. Atonal ist Sascha Muhteschem im Roman Gefährliche Verwandtschaft, ein Mehrsprachler, der seine auseinanderdriftenden Geschichtsstränge wieder aneinander zu koppeln versucht. Sascha hat deutsche Großeltern, die als ,Kulturjuden“ während der NS-Herrschaft Zuflucht in der Türkei finden mussten, und einen türkischen Großvater, der an der Gründung der türkischen Republik und am Völkermord an den Armeniern mitwirkte. Eine schwer erfassbare transnationale Identität, die heute dabei ist, zur Norm zu werden: „Es gibt viel mehr lose und wechselnde Bindungen als früher, ,Luftidentitäten“, die ihre Ressourcen aus transnationalen Zugehörigkeiten beziehen. “ (Șenocak 2011, 22). Das Atonale soll also nicht negativ bewertet, sondern als Chance wahrgenommen werden, als die Chance des Deutschwerdens, das kein loses Nebeneinander, kein „berührungslose[s] Nebeneinander von Kulturen und Lebensanschauungen“ (Șenocak 1993, 11) darstellt, sondern ein bewusstes, engagiertes Miteinander.

Engagement, politisches wie zivilgesellschaftliches Engagement, forderte nämlich das zweite weltgeschichtliche Ereignis, die Terrorattentate vom 11. September 2001. Über Nacht gerieten Bürger moslemischer Konfession unter Generalverdacht. Über Nacht wurde ,Moslem‘ Synonym für ,Terrorist‘, womit die verschiedenen Islamströmungen verkannt werden. Auch nationale Unterschiede zwischen den moslemischen Hinzugekommenen wurden dabei verwischt. In seiner Schrift Deutschsein. Eine Aufklärungsschrift (2011) bemängelt Șenocak die „,ürkisch-arabische Bindestrichidentität“, die in Deutschland durch „eine Überbetonung der muslimischen Identität“ konstruiert wurde (Șenocak 2011, 87). Dieses Konstrukt beweist, wie nun Religion zum nationalen Identitätsmarker einer Gruppe erhoben wird, und hört sich im Umkehrschluss wie der Wille zu einer homogenen deutschen Identität an.

2 Seinem Essayband Atlas des tropischen Deutschland geht folgendes Zitat aus Elias Canettis Masse und Macht (1976) voran: „Das Massensymbol der Deutschen war das Heer. Aber das Heer war mehr als das Heer: es war der marschierende Wald. In keinem modernen Lande der Welt ist das Waldgefühl so lebendig geblieben wie in Deutschland. Das Rigide und Parallele der aufrechtstehenden Bäume, ihre Dichte und ihre Zahl erfüllt das Herz des Deutschen mit tiefer und geheimnisvoller Freude. Er sucht den Wald, in dem seine Vorfahren gelebt haben, noch heute gern auf und fühlt sich eins mit Bäumen. Ihre Sauberkeit und Abgegrenztheit gegeneinander, die Betonung des Vertikalen, unterscheidet diesen Wald von dem tropischen, wo Schlinggewächse in jeder Richtung durcheinanderwachsen. Im tropischen Wald verliert sich das Auge in der Nähe, es ist eine chaotische, ungegliederte Masse, auf eine bunteste Weise belebt, die jedes Gefühl von Regel und gleichmäßiger Wiederholung ausschließt.“ (Șenocak 1993, 5). 
Nicht nur ethnisiert fühlte sich Zafer Șenocak, sondern auch konfessionalisiert, ,muslimifiziert‘ (vgl. Sezgin 2010). Der Autor, der seine Andersartigkeit nie als Stigma erlebte, sondern als Bestandteil der ,neudeutschen Gesellschaft', musste Farbe bekennen. Er resignierte aber nicht. Er lehnte sowohl die Assimilierung als auch den identitären Rückzug ab. Als Intellektuellem kam ihm die Aufgabe zu, gerade durch die Betonung seiner individuellen Einzigartigkeit zum Sprachrohr der „neudeutschen“ Gesellschaft zu werden.

Șenocak führt nun einen Zweifrontenkampf, wobei er sich immer wieder auf die deutsche Aufklärung und die türkische Mystik bezieht. Beiden Bewegungen ist Toleranz gemeinsam. Als Vorbilder gelten vor allem Lessing, Kant und Yunus Emre. Șenocak warnt einerseits vor pauschalisierenden Urteilen, die man von der Mehrheitsgesellschaft immer wieder zu hören bekommt, vor der wachsenden Essenzialisierung der Moslems in Deutschland. Diese Vorurteile werden auch durch den Mediendiskurs genährt, der bei der Ethnisierung der Kriminalität, der Bildungsferne - „Ein Türke [...] geht nicht in die Oper“ (Șenocak 1993, 22), der Transferabhängigkeit usw. mitwirkt. Das Individuelle wird dabei völlig negiert. Andererseits sagt Șenocak den moslemischen Fundamentalisten den Kampf an. Sie werden seiner Meinung nach durch den Mangel an ideellen Auseinandersetzungen im Islam und mit dem Islam, durch den zur Regel erhobenen Grundsatz der Textgläubigkeit auf Kosten der individuellen Auslegung und einer allegorischen Lektüre des Korans in die Irre geführt und führen die anderen Moslems in die Irre. Șenocak plädiert für einen aufgeklärten Islam in einem säkularisierten Staat: Religion sei reine Privatsache. Er fordert die Moslems dazu auf, sich die deutsche Aufklärung und die türkische Mystik zum Vorbild zu nehmen und keine Debatte zu scheuen: „[Die Muslime] müssen endlich anfangen, ihre Tradition kritisch zu betrachten, freie Meinungsäußerung nicht nur zu dulden, sondern auch zu fördern“ (Șenocak 1993, 18). Umso mehr, als der Islam an eine frühere aufklärerische Tradition anknüpfen kann:

Längst überfällig ist heute die Wiederaufnahme und Entwicklung jenes kritisch aufklärerischen Geistes, der vom 9. bis zum 13. das morgenländische Denken bestimmte, eine hohe Zivilisation aufblühen ließ und das europäische Mittelalter auf dem Wege zur Neuzeit entscheidend geprägt hat. (Șenocak 1993, 19)

In einem Welt-Artikel vom 22. November 2015, „Von der Einsamkeit der aufgeklärten Muslime“, geht er mit dem islamischen Fundamentalismus und dem türkisch-arabischen Autokratentum ins Gericht, die heutzutage den Islam entstellen und die aufgeklärten Moslems ausschließen und sogar gewaltsam unterdrücken. Beiden Seiten wirft er Einseitigkeit, Aus- und Abgrenzung und Streben nach einer homogenen Gemeinschaft vor, in die der Andere nicht gehört. 
Islamische Fundamentalisten genauso wie westliche Nationalisten erschreckt das Hybride, weil es die Grenzen des Vertrauten sprengt. Beide Bewegungen stünden nämlich einander sehr nahe, wie er es im Mai 2016 in einem Interview für Deutschlandradio Kultur, „Nationale Identität, die Europa spalten will“, unterstreicht:

Sie gehören zusammen und sind Partner auf dem Weg in ein neues Europa, das von Nationalgefühlen geprägt ist. Die stramm konservativen Muslime der Türkei und die alternativen Rechten Europas sind die beiden Seiten einer Medaille. Sie werden sich rhetorisch gegenseitig aufschaukeln und am Ende alle Brücken einreißen, nicht nur zwischen einem christlichen Abendland und einem islamischen Morgenland, sondern zwischen gesellschaftlichen Gruppen quer über den Kontinent, in Deutschland nicht anders als in der Türkei. (Șenocak 2016)

Beiden Seiten wirft er auch vor, die langwierigen Bemühungen um gegenseitige Anerkennung zunichtezumachen. Ein guter Grund, sich immer mehr für die Umsetzung der „Vielvölkerrepublik“ (Șenocak 2011, 55), wie er auch das nun heterogene, hybride, tropische, atonale Deutschland nennt, einzusetzen. Mit „Republik“ meint er die Werte, auf denen die Bundesrepublik Deutschland gegründet wurde, mit denen sie sich in die Tradition der Aufklärung und des Humanismus stellte und auch heute noch stellt. In dieses Konzept passt auch die Toleranz des Sufismus, dem eine integrative Funktion zukommt. Deswegen ruft er die Deutschen, die „Neuen Deutschen“ und auch die Nichtdeutschen zur Überwindung des ethnisch-religiös nationalstaatlichen Eingebundenseins auf und verlangt von ihnen mehr Willen zum Miteinander. Von ihnen erwartet er, dass sie eine Willensgemeinschaft bilden, die die frühere Schicksalsgemeinschaft ablösen soll: „Die türkische Jugend Deutschlands“ dürfe „sich nicht an die Phantasmagorie der verlorenen Heimat klammern“ und „althergebrachte Identitäten mumifizieren“ (Șenocak 1993, 13-15), und die hiesige Gesellschaft solle den eigenen Wandel berücksichtigen. „Heimat ist kein Schicksal“, so Zafer Șenocak in einem Deutschlandfunk-Beitrag vom 2. Februar 2017.

\section{Literaturverzeichnis}

Amodeo, Immocolata, und Heidrun Hörner, und Christiane Kiemle (Hg). Literatur ohne Grenzen. Interkulturelle Gegenwartsliteratur in Deutschland - Porträts und Positionen. Sulzbach/ Taunus: Ulrike Helmer, 2009. Arnold, Heinz Ludwig (Hg.). Literatur und Migration. München: text+kritik, 2006. Chiellino, Carmine (Hg.). Interkulturelle Literatur in Deutschland. Ein Handbuch. Stuttgart und Weimar: Metzler, 2000. 
Genton, François, und Susanne Berthier-Foglar (Hg). L'Allemagne et les migrants. Théories, stratégies et regards croisés sur une réalité complexe. Paris: L'Harmattan, 2018.

Glissant, Edouard. L'imaginaire des langues. Paris: Gallimard, 2010.

Glissant, Edouard. Introduction à une poétique du divers. Paris: Gallimard, 1996.

Glissant, Edouard. Tout-monde. Paris: Gallimard, 1995.

Goffman, Erving. Stigma. Über Techniken der Bewältigung beschädigter Identität. Frankfurt/M.: Suhrkamp, 2010.

Grimm, Günter E., und Klaus-Michael Bogdal (Hg.). Einführung in die interkulturelle Literatur. Darmstadt: WBG, 2015.

Hofmann, Michael. Interkulturelle Literaturwissenschaft. Paderborn: UTB, 2006.

Münkler, Herfried, und Marina Münkler. Die neuen Deutschen: Ein Land vor seiner Zukunft. Berlin: Rowohlt, 2016.

Pörksen, Uwe, und Bernd Busch (Hg.). Eingezogen in die Sprache, angekommen in der Literatur. Positionen des Schreibens in unserem Einwanderungsland. Göttingen: Wallstein, 2008.

Schaffernicht, Christian (Hg.). Zu Hause in der Fremde. Ein Ausländerbuch. Fischerhude: Atelier im Bauernhaus,1981.

Șenocak, Zafer. Atlas des tropischen Deutschland. Berlin: Babel, 1993.

Șenocak, Zafer. Deutschsein. Eine Aufklärungsschrift. Hamburg: Körber-Stiftung, 2011.

Șenocak, Zafer. „Drei Kontinente“. Das Argument. Zeitschrift für Philosophie und Sozialwissenschaften 219 (1997): 157.

Șenocak, Zafer. Der Erottomane. München: Babel, 1999.

Șenocak, Zafer. Gefährliche Verwandtschaft. München: Babel 1998.

Șenocak, Zafer. „Heimat ist kein Schicksal“. Deutschlandfunk Kultur, 2. Februar 2017.

Șenocak, Zafer. In deinen Worten: Mutmaßungen über den Glauben meines Vaters. München: Babel, 2016.

Șenocak, Zafer. das land hinter den buchstaben. Deutschland und der Islam im Umbruch. München: Babel, 2006.

Șenocak, Zafer. „Nationale Identität, die Europa spalten will“. Deutschlandfunk Kultur, 4. Mai 2016.

Șenocak, Zafer. Pavillon. Berlin: Dyageli, 2009.

Șenocak, Zafer. Die Prärie. Hamburg: Rotbuch, 1997.

Șenocak, Zafer. „Von der Einsamkeit der aufgeklärten Muslime“. Die Welt, 22. November 2015. https://www.welt.de/debatte/kommentare/article149117239/Von-der-Einsamkeit-deraufgeklaerten-Muslime.html.

Șenocak, Zafer. War Hitler Araber? Irreführungen an den Rand Europas. Berlin: Babel,onlineQuelle: 1994.

Șenocak, Zafer. „,Willkommen heißen“, ,anerkennen“, ,integrieren“ - oder einfach mal machen!“ Diskussionsrunde am 29. November 2013. Ankommen in der Gesellschaft der Vielfalt. Hg. Bernadette Schwarz-Boenneke. Freiburg, Basel, Wien: Herder, 2014.

Șenocak, Zafer. Zungenentfernung. Bericht aus der Quarantänestation. München: Babel, 2001.

Sezgin, Hilal. „Deutschland schafft mich ab“. Die Zeit, 3. September 2010. https://www.zeit. de/2010/36/Muslimifizierung.

Uysal-Ünalan, Saniye. Interkulturelle Begegnungsräume. Neue Identitätskonstruktionen. Online-Quelle in der türkisch-deutschen Gegenwartsliteratur. Würzburg: Königshausen \& Neumann, 2013.

Warakomska, Anna, und Mehmet Öztürk (Hg.). Man hat Arbeitskräfte gerufen, ... es kamen Schriftsteller. Die Migranten und ihre Literaturen. Frankfurt/M.: Peter Lang, 2015. 
Marie-Noëlle Faure, Professeur de chaire supérieure in literarischen Vorbereitungsklassen BL (Schwerpunkt Kultur- und Sozialwissenschaft) am Lycée Henri IV in Paris. Unterrichtet dort deutsche Geschichte und Kultur. Letzte Veröffentlichungen: „,Géographie personnelle“ et identité allemande chez Zafer Șenocak“. L'Allemagne et les migrants. Théories, stratégies et regards croisés sur une réalité complexe. Hg. François Genton und Susanne Berthier-Foglar. Paris: L'Harmattan, 2018. 209-224; Luther et la Réforme. Paris: Editions Studyrama, 2016; „Von der Chamissoliteratur zur Ankunftsliteratur. Interkulturelle Literatur und Neusbestimmung des Deutschseins“. Man hat Arbeitskräfte gerufen, ... es kamen Schriftsteller. Die Migranten und ihre Literaturen. Hg. Anna Warakomska und Mehmet Oztürk. Frankfurt/M.: Peter Lang, 2015. 41-56. 